\title{
Protease-activated quantum dot probes based on fluorescence resonance energy transfer
}

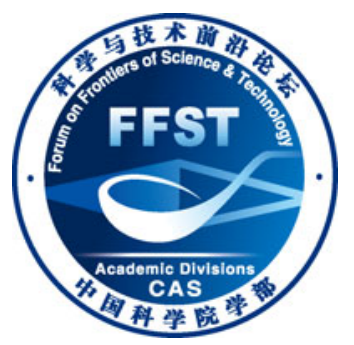

\author{
LI Xin, XUE Bing, LI Yang \& GU YueQing* \\ Department of Biomedical Engineering, School of Life Science and Technology, China Pharmaceutical University, Nanjing 210009, China
}

Received December 24, 2012; accepted January10, 2013; published online May 3, 2013

\begin{abstract}
A novel sensing system based on fluorescence resonance energy transfer (FRET) between CdTe quantum dots (QDs) and Rhodamine $\mathrm{B}(\mathrm{RB})$ was established for the detection of matrix metalloproteinases (MMOL/LPs). In this system, 535-nm-emitting quantum dots (QDs) were bound to Rhodamine B (RB) via a MMOL/LP-specific peptide. A 76\% reduction in luminescence was achieved because of FRET. Release of RBs by peptide cleavage restores radiative QD photoluminescence. Initial studies observed a $73 \%$ rise in luminescence over $60 \mathrm{~min}$. The design platform of the nanosensor is flexible and can be fine-tuned for a wide array of applications such as the detection of biomarkers, early diagnosis of disease, and monitoring therapeutic efficacy simply by changing the sequence of the peptide linker.
\end{abstract}

quantum dots, fluorescence resonance energy transfer, matrix metalloproteinases, proteolytic activity, bioimaging

Citation: $\quad$ Li X, Xue B, Li Y, et al. Protease-activated quantum dot probes based on fluorescence resonance energy transfer. Chin Sci Bull, 2013, 58: 2657-2662, doi: $10.1007 / \mathrm{s} 11434-013-5712-7$

Homogeneous semiconductor nanocrystals or quantum dots (QDs) in the 2-6 $\mathrm{nm}$ size range are of considerable current interest, not only because of their unique size-dependent properties, but also because of their dimensional similarities with biological macromolecules (e.g. proteins and nucleic acids) [1,2]. These similarities could allow an integration of nanotechnology and biology, make them potential candidates as a platform for biorecognition events. The modulation of QD luminescence provides the opportunity for the transduction of these events via fluorescence resonance energy transfer (FRET), electrochemiluminescence (ECL), charge transfer quenching, and bioluminescence resonance energy transfer (BRET) [3-5].

Up to now, much attention has been paid to FRET method for in vitro and in vivo assays of proteases due to its simplicity, rapidness, sensitivity and reproducibility [6]. QDs are excellent donors in FRET because of their numerously advantageous features. First, QDs provide the opportunity for the interaction of a single donor with multiple acceptors

*Corresponding author (email: guyueqingsubmission@ hotmail.com) via assembly at the QD surface. Next, since the quantum yield of QDs is generally high, the size-tunable photoluminescence (PL) of QDs provides large donor-acceptor spectral overlap integral and a possibility for maximizing the Förster distance. Another advantage is afforded by the broad absorption spectrum of QDs, which make it easier to work far from wavelengths associated with acceptor dye transitions. Furthermore, the high molar absorption coefficients and potentially high quantum yields of QDs also allow the use of lower intensity excitation, further minimizing the direct excitation of acceptors and photobleaching rates [7-10].

It is now evident that matrix metalloproteinases (MMOL/ LPs), as the most prominent family of proteinases associated with tumorigenesis, function more complex than modulators of the tumor microenvironment [11-13]. Rather, they also take a role in extracellular matrix turnover and cancer cell migration, MMOL/LPs regulate signaling pathways that control cell growth, angiogenesis, or inflammol/Lation, and may even work in a nonproteolytic manner [14]. We designed a probe that are only based on substrates might be 
limited by their overall lack of region specificity. The peptide sequence present in interstitial collagen targeted by a variety of proteases including MMOL/LP-2, -9 , and -14 , which can be used to detect MMOL/LP activity predominantly at the leading edges of migrating tumor cells [15].

This probe consists of a QD with a green fluorescence emission as a donor of FRET, an organic dye Rhodamine B (RB) as a fluorescent receptor, whith connected with a MMOL/LP-specific peptide [16]. It was hypothesized that QDs labeled activatable probes induce a higher specificity and sensitivity of the probe in vivo, since the nanoparticles can deliver the probe effectively to the disease sites (i.e. tumor region) by the enhanced permeation retention effect and because the peptide substrate-mediated fluorescence labeling of the QDs can be strongly quenched [17]. When the probe is exposed to the specific MMOL/LP of interest, cleavage of the substrate occurs due to specific substrate recognition by the MMOL/LPs; this is manifest in the form of a pronounced fluorescence signal recovery due to dequenching of the dye (Figure 1).

\section{Materials and methods}

\subsection{Materials and chemicals}

Tellurium powder, sodium borohydride, absolute ethanol, chloroform, isoamyl alcohol, $\mathrm{CdCl}_{2} \cdot 2.5 \mathrm{H}_{2} \mathrm{O}, \mathrm{NaBH}_{4}, \mathrm{~N}$ acetyl-L-cysteine (NAC), N-hydroxysuccinimide (NHS), 1-[3-(dimethylamino)prop-yl]-3-ethylcarbodiimide-methiodide (EDC), penicillinsodiumhydr-oxide and sulfuric acid were of analytical grade. Red-free RPMI 1640 and DMEM (Sangon Biotech, Shanghai, China). Fetal bovine serum (FBS), streptomycin, penicillin, trypsin-EDTA were purchased from Gibco (Life Technologies, Shanghai, China). MMOL/LPsspecific peptide linked with Rhodamine B (RB) was purchased from Apetide (Chutai Bio-technology, Shanghai, China). Redistilled water was used in all experiments.

\subsection{Quantum dot synthesis}

NAC-stabilized CdTe quantum dots were synthesized according to the reported method [18]. Firstly, the sodium hydrogen telluride (NaHTe) was prepared as follows. Under $\mathrm{N}_{2}$ atmosphere, $\mathrm{NaBH}_{4}$ was used to react with tellurium at a molar ratio of 3:1 in redistilled water/absolute ethanol. Subsequently, the fresh NaHTe solution was added to the

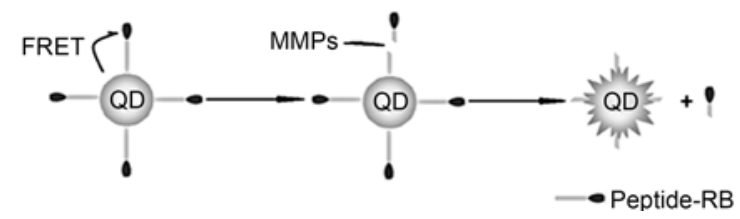

Figure 1 A schematic of FRET-based MMOL/LP-2 detection method using QDs-peptide-RB nanoassembly. prepared precursor solution $(n(\mathrm{Cd})=0.2 \mathrm{mmol} / \mathrm{L}, n(\mathrm{NAC})=$ $0.8 \mathrm{mmol} / \mathrm{L}, \mathrm{pH} 8.5$ ) under vigorous stirring in $\mathrm{N}_{2}$ atmosphere. The feed molar ratio of $\mathrm{Cd}: \mathrm{Te}: \mathrm{NAC}$ was fixed at $1.0: 0.5: 4$. Then it was refluxed at $95^{\circ} \mathrm{C}$. The QDs with different fluorescence were synthesized under different refluxing time separately. The resulting CdTe QDs were precipitated with cold 2-propanol and then isolated by centrifugation and decantation to remove excess Cd-NAC complexes.

\subsection{Bioconjugation of QDs and peptide-RB}

QDs dissolved in deionized water $(1 \mathrm{mg} / \mathrm{mL})$ were activated with EDC and NHS to form an active ester leaving group. The N-terminus of the synthesized peptide-RB was then covalently linked to the QD at the active ester site to form an amide. Peptide activation of the C-terminus was prevented by reacting residual EDC with $\beta$-mercaptoethanol prior to peptide addition. At the coupling reaction, peptide was added at $\mathrm{pH} 7$ in a series of feed molar ratio of $\mathrm{QD}: \mathrm{RB}$ $(1: 3,1: 4,1: 5,1: 6$ and $1: 7)$ to ensure properly coupling onto the QD. The reaction was allowed to proceed overnight in the dark at room temperature. The solution was then dialyzed with 3500 MWCO (Spectrum, America) cellulose ester membrane to remove any unreacted peptide-RB or byproducts. Finally, store them in $4^{\circ} \mathrm{C}$ refrigerator after freeze-drying and weighing.

\subsection{Characterization of the probe}

Dissolve the probes in deionized water $(2 \mathrm{mg} / \mathrm{mL})$. All measurements were performed at $\mathrm{pH} 4.0$ and room temperature with a $200 \mu \mathrm{L}$ quartz stoppered cuvette (Wanqing chemical classware instrument, Nanjing, China) to prevent evaporation. Absorption and photoluminescence spectra of the solution were measured by 754-PC UV-Vis spectrophotometer (Jinghua Technological Instrument Corporation, Shanghai, China) and Lengguang F96 Fluorescence spectrophotometer $\left(\lambda_{\mathrm{ex}}=370 \mathrm{~nm}\right)$. Transmission electron microscopy was performed to investigate the morphology of CdTe QDs and probes alloyed on JEOL JEM-2100 electron microscopes.

Fourier transform infrared spectroscopy (FTIR) tests were conducted on a Impact 410 FTIR spectrometer (Nicolet, America). Samples were made into films by mixing them with $\mathrm{KBr}$.

\subsection{Preparation of enzyme solution}

Five cell lines (MCF-7, HepG2, Bel-7402, A549 and MDAMB-231) were used for MMOL/LP-2 expression evaluation by reverse transcriptase PCR. Briefly, total RNA was extracted from freshly isolated cancer cells using Rneasy Kit. RNA $(3 \mu \mathrm{g})$ from each cancer cell line was converted into cDNA with Superscript III reverse transcriptase. Subsequently, $1.0 \mu \mathrm{L}$ cDNA was used for PCR amplification with 
MMOL/LP-2 primers (sense: 5'-GGAGCATGGCGATGGATA-3', antisense: 5'-CAGTGGACATGGCGGTCT-3'). The product length was $491 \mathrm{bp}$. The product $(5 \mu \mathrm{L})$ was used for $2 \%$ agarose gel electrophoresis.

MDA-MB-231 breast cancer cells over-expressing MMOL/ LPs (MMOL/LP+) and MCF-7 breast cancer cells with low expression of MMOL/LPs (MMOL/LP-) were used to assess the specificity of the probe. Each cell line was grown in phenol red-free DMEM and RPMI 1640 solution that included $10 \%$ fetal bovine serum, $2 \mathrm{mmol} / \mathrm{L}$ L-glutamine and $1 \%$ penicillin-strep-tomycin under standard conditions at $37^{\circ} \mathrm{C}$ and a humidified atmosphere containing $5 \%$ $\mathrm{CO}_{2}$ and $95 \%$ air for at least $18 \mathrm{~h}[19,20]$. After the cell culture to logarithmic phase, the medium was removed and stored in $4^{\circ} \mathrm{C}$ refrigerator.

\subsection{Activation of the probe}

QD probe was added to the MDA-MB-231 medium solution at a final concentration of $0.8,1,1.2,1.4,1.6,1.8,2 \mathrm{mg} / \mathrm{mL}$ and stirred for $2 \mathrm{~h}$. Time-integrated photoluminescence was measured before and after cleavage of the probe to observe recovery of $\mathrm{RB}$ at $\mathrm{pH} 4$. Control samples (QD probe with MCF-7 medium solution at a concentration of $2 \mathrm{mg} / \mathrm{mL}$ ) were monitored simultaneously.

\section{Results and discussion}

\subsection{Confirmation of the FRET system}

Under optimized conditions, the fluorescence spectra of CdTe QDs were tunable in the range of 525-650 nm by changing the refluxing time. The QDs refluxed 69 min with emission maximum at 535 were used in the present study. And their quantum yields were measured to be $63 \%-70 \%$.

Fluorescent material acted as the donor and receptor needs to meet some certain conditions before they can produce FRET, such as the distance must be less than $10 \mathrm{~nm}$, a dipole relative orientation, and the most important, the donor fluorescence emission and absorption of the receptor have a large overlap [6]. Although a variety of organic dyes can be used as a FRET fluorescent donor-receptor pairs, we contact RB and QD by the peptide at each end, so that it satisfies the conditions of less than $10 \mathrm{~nm}$. Figure 2 shows that the emission of QDs overlaps well with the absorption of RB labeled peptides, and their emission spectra overlap very little, suggesting an efficient FRET between them.

We further analyzed the UV absorption of the FRET probe (Figure 3). The absorption spectral of RB-coupled peptides showed the characteristic absorption peaks, which could be used for RB (555 $\mathrm{nm})$. As QDs have a wide range of 250-500 nm UV absorption which overlap with original peptide-RB, there was only an increase from 250 to $500 \mathrm{~nm}$ observed at the absorption spectral of QD probe, but not the characteristic peaks of QDs. And the characteristic absorption

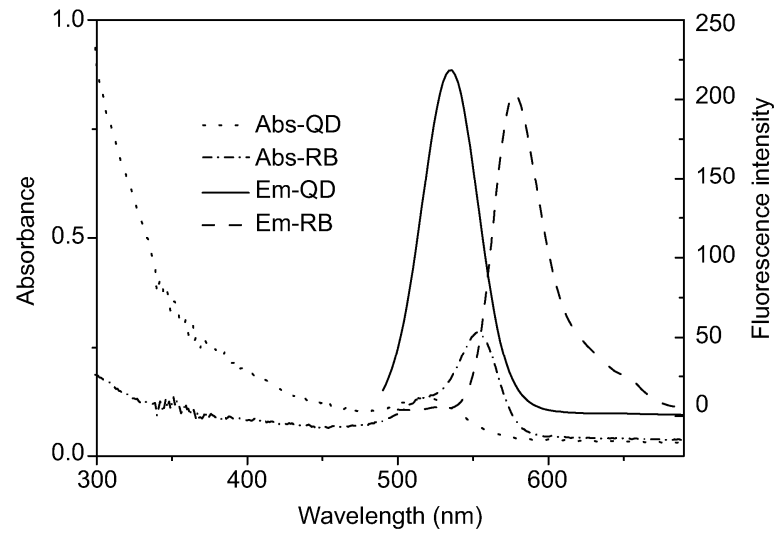

Figure 2 UV-Vis absorption and fluorescence emission spectra of NACcapped QDs and RB.

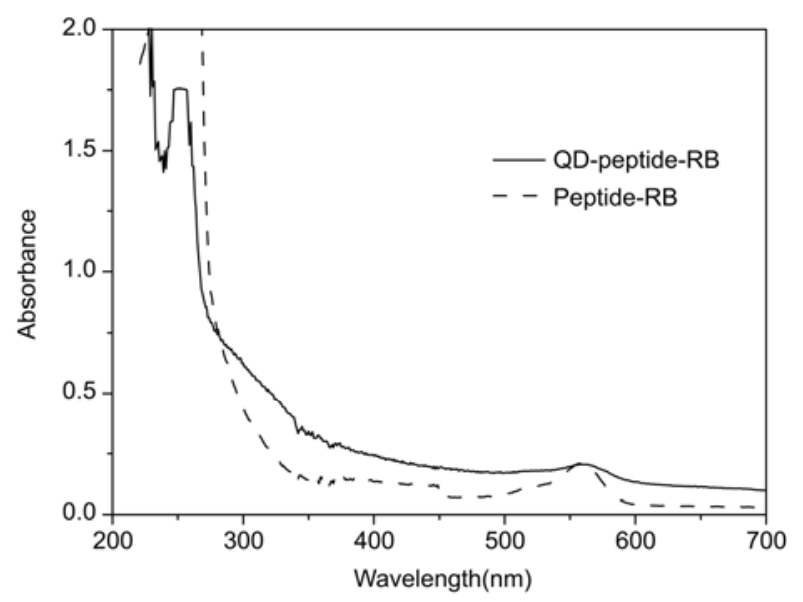

Figure 3 UV-Vis absorption spectral of peptide-RB and QDs-peptideRB UV-Vis absorption spectral of the FRET-based probes.

peaks could be used for confirming peptides $(280 \mathrm{~nm})$. Because of the dialysis of small molecular (peptide-RB), we could determine the peptide-RB ultimately connected with the quantum dots.

FTIR spectroscopy is helpful to confirming the conjugation of CdTe QD to peptide. In Figure 4, the FTIR spectra of NAC-capped CdTe QDs show a peak at $3443 \mathrm{~cm}^{-1}$, this particular band can be assigned to $-\mathrm{OH}$ and $-\mathrm{NH}$ stretching mode of NAC. The peaks at 1208 and $1140 \mathrm{~cm}^{-1}$ correspond to the stretching vibration of $\mathrm{C}-\mathrm{N}$. The narrower peak at $1682 \mathrm{~cm}^{-1}$ is typical of $\mathrm{C}=\mathrm{O}$, and the observed shift could be due to the coordination of the $\mathrm{Cd}$ atoms present on the surface of CdTe QDs.

Figure 5 describes the FTIR spectra of QD-peptide-RB. It can be noticed that there is a broad absorption peak at $3396 \mathrm{~cm}^{-1}$, characteristic of the stretch vibration of $\mathrm{O}-\mathrm{H}$ as well as $\mathrm{N}-\mathrm{H}$. The peaks at 1598 and $1290 \mathrm{~cm}^{-1}$ are assigned to $\mathrm{CONH}-$, resulting from the conjugation of QD and peptide. While the band at $1395 \mathrm{~cm}^{-1}$, more than likely the overlap of two individual peaks, is ascribed to the symmol/ Letrical deformation of $\mathrm{CH}_{2}$ and $\mathrm{CH}_{3}$ come from the peptide. 


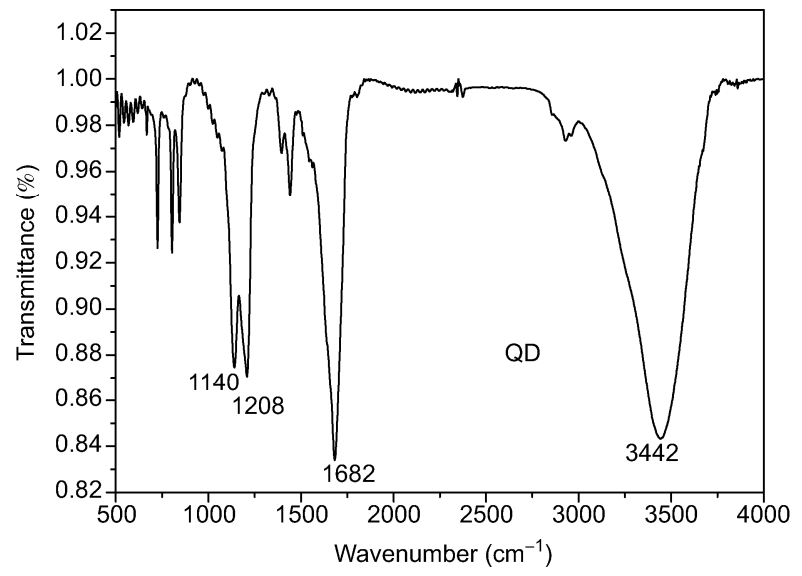

Figure 4 FTIR spectra of QDs.

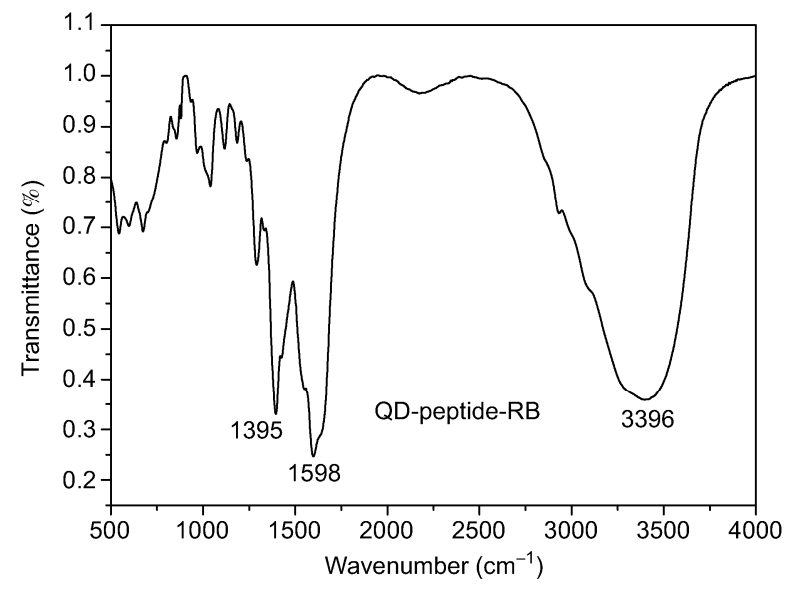

Figure 5 FTIR spectra of QD-peptide-RB.

\subsection{Optical properties of QDs probe}

Figure 6 depicts the fluorescence of the RBs that was stimulated by QDs through FRET. Activated ester solution was added to the reaction system with different contents of QDs. As a result, RB fluorescence gradually increased with the increase of the the reaction feed ratio of QDs. Spectroscopic analysis of the QD-functionalized RB indicated that approximately 6 units of RB associated with each particle were the most effective. Further increase in the QDs activated ester decreased the FRET efficiency. This may be due to an excessive number of QDs not connected with the peptide, which cannot yield RB energy transfer phenomenon, but need excitation energy to stimulate, resulting in reduction of QDs fluorescence intensity using for FRET. As the $\mathrm{pH}$ of solution was acid, the characteristic peaks of QDs at $535 \mathrm{~nm}$ was not shown [21]. But as soon as the $\mathrm{pH}$ was adjusted to 8, fluorescence of the QDs occurred. Figure 7, which depicts the luminescence of the QDs stimulated by RBs through FRET, proves the same reaction ratio.

QD-peptide-RB luminescence performed a 76\% exaltation of signal compared to unreacted peptide-RB (Figure 8). It

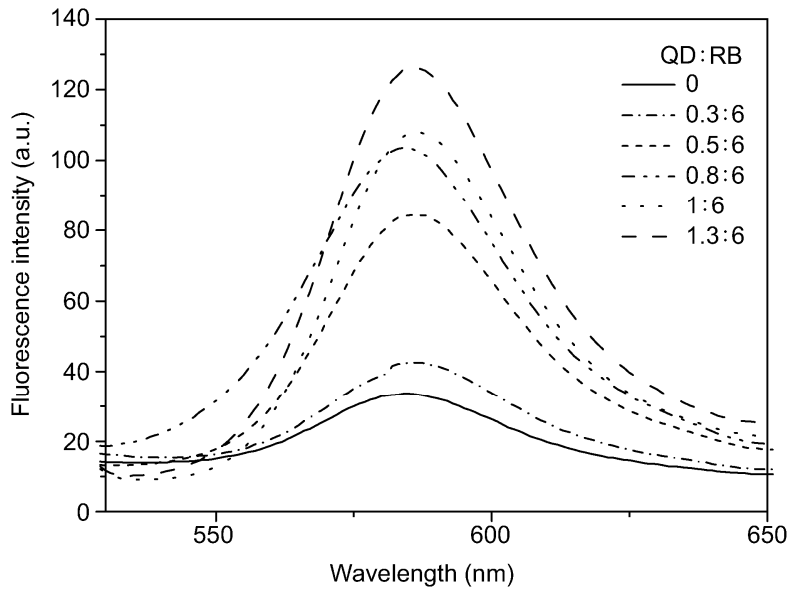

Figure 6 Fluorescence spectra of QD-peptide-RB probes with different feed molar ratio of QD:RB.

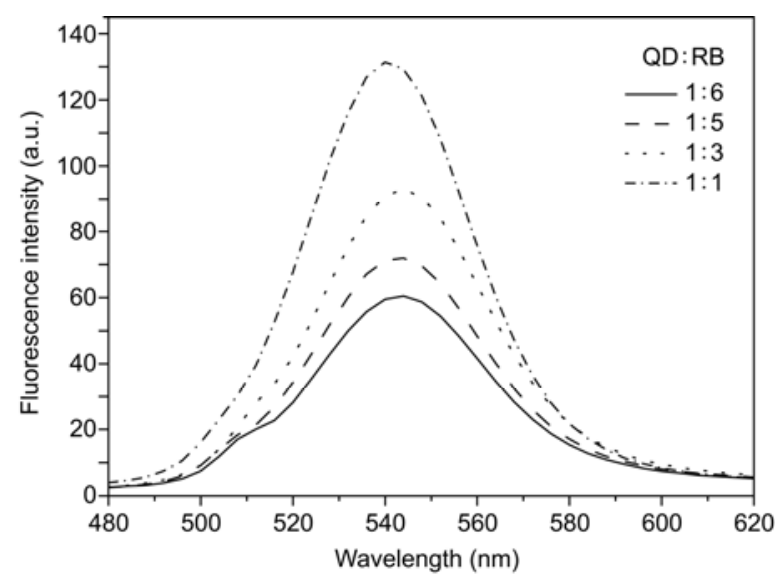

Figure 7 Fluorescence spectra of QD-peptide-RB probes with different feed molar ratio of QD: RB.

was obvious that $\mathrm{QD}$ and $\mathrm{RB}$ were within a short distance linked by the peptide, which induced an efficient energy transfer and consequently resulted in the fluorescence stimulating of the RB.

\subsection{Morphological characterization}

Transmission electron microscopy (TEM) was used to investigate the morphology and crystal structure of CdTe QDs and QD probes. Figure 9(c) shows a low-magnification TEM image of the prepared QDs, indicating that these nanocrystals were close to spherical with excellent polydisperisity and average size was about $3.5 \mathrm{~nm}$. The average size of QD probes was $3.8 \mathrm{~nm}$ (Figure 9(a)) which implied some connection with substrates. Further analysis of the crystalline structures was performed by high-resolution TEM images (Figure 9(b),(d)). QDs presented clear intact crystal lattice lines, and their crystal structures could be attributed to the cubic (zinc blende) phase consistent. Thus, the structure of QDs did not change after the bioconjugation. 


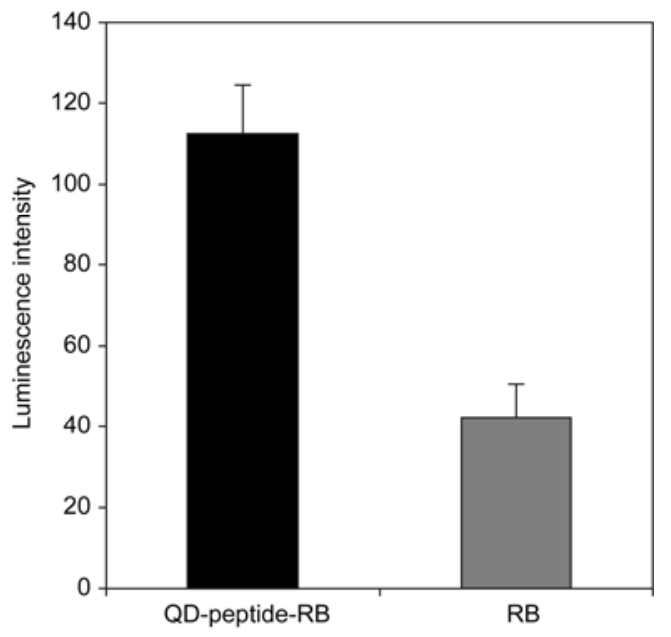

Figure 8 This chart demonstrates the exaltation in peptide-RB luminescence after covalent bonding of QDs. Fluorescence intensity at $585 \mathrm{~nm}$ was measured.

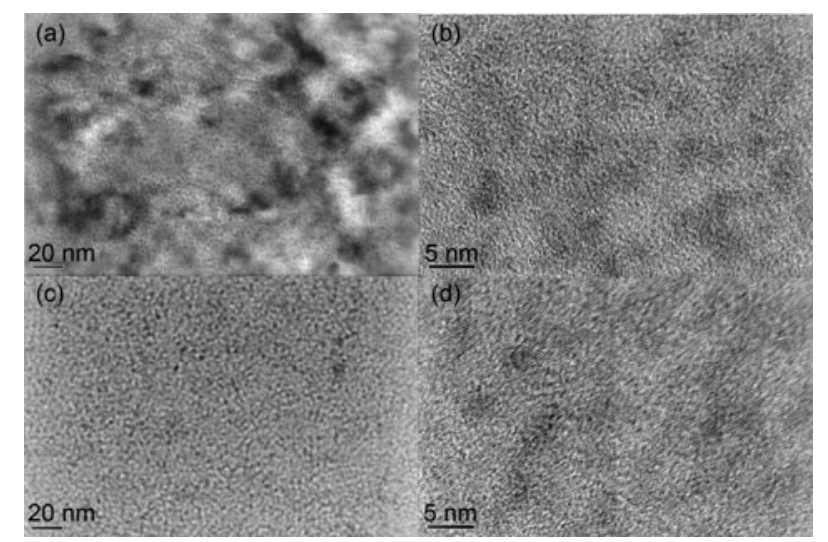

Figure 9 Low-magnification TEM image (a) and HR-TEM image (b) of CdTe QDs with PL emission peak at $535 \mathrm{~nm}$; low-magnification TEM image (c) and HR-TEM image (d) of QD-peptide-RB probes.

\subsection{Fluorescence recovery of QDs}

Figure 10 shows the expression of MMOL/LP-2 in a number of cells. Real-time PCR analysis revealed that the MMOL/LP-2 mRNA expression was markedly different in cells, a high level expression was observed in MDA-MB231; low level in MCF-7, HepG2, Bel-7402 and A549, which previous findings have indicated.

Since MMOL/LPs were enzyme of secretory, they would exist in the medium if expressed. Luminescence of each sample in the activation of the probe was measured at the following time points: 0, 10, 20, 30, 40 and $60 \mathrm{~min}$. Figure 11 illustrates the spectral profile of reduced RB luminescence over time. And there was very little autofluorescence at $585 \mathrm{~nm}$ from enzyme solution. A reduction in luminescence over time of the probe with MMOL/LPs was observed in Figure 12. At $60 \mathrm{~min}$, an average luminescence lower of $73 \%$ was performed. The control group containing inacti-

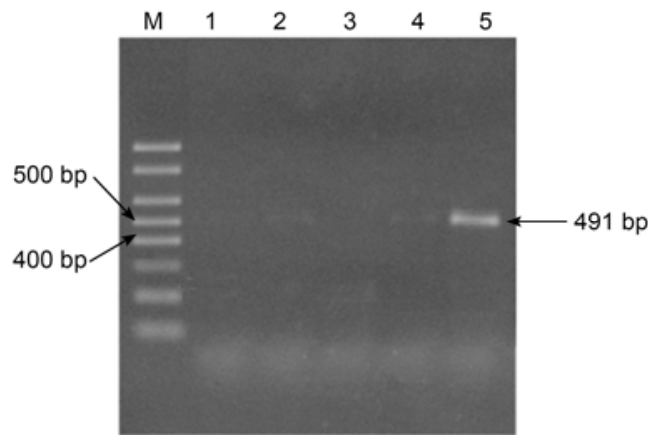

Figure 10 RT-PCR screening of MMOL/LP-2 present in five diffrernt cells. M, DNA marker; 1, MCF-7; 2, HepG2; 3, Bel-7402; 4, A549; 5, MDA-MB-231.

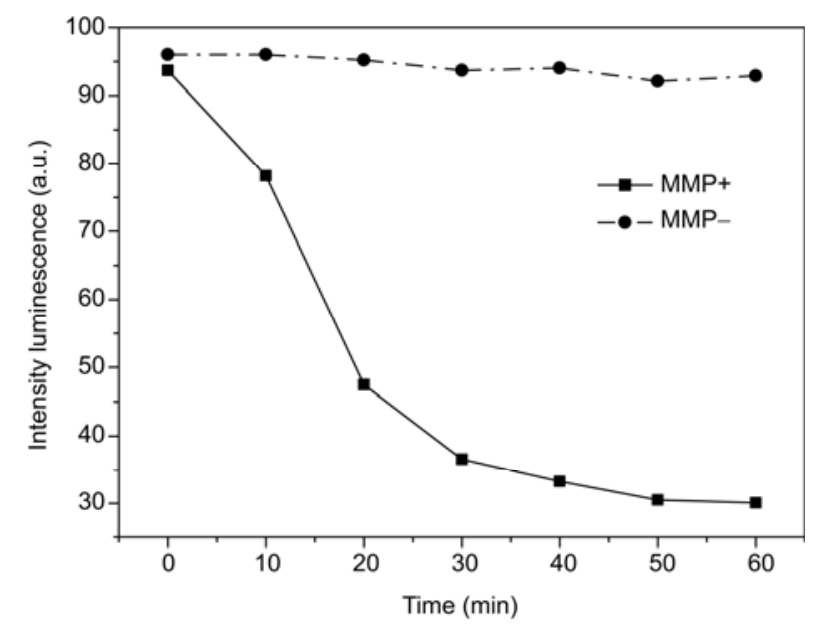

Figure 11 Plot of probes activation with MMOL/LP+(MDA-MB-231 medium) solution and MMOL/LP-(MCF-7 medium) solution increased luminescence over time.

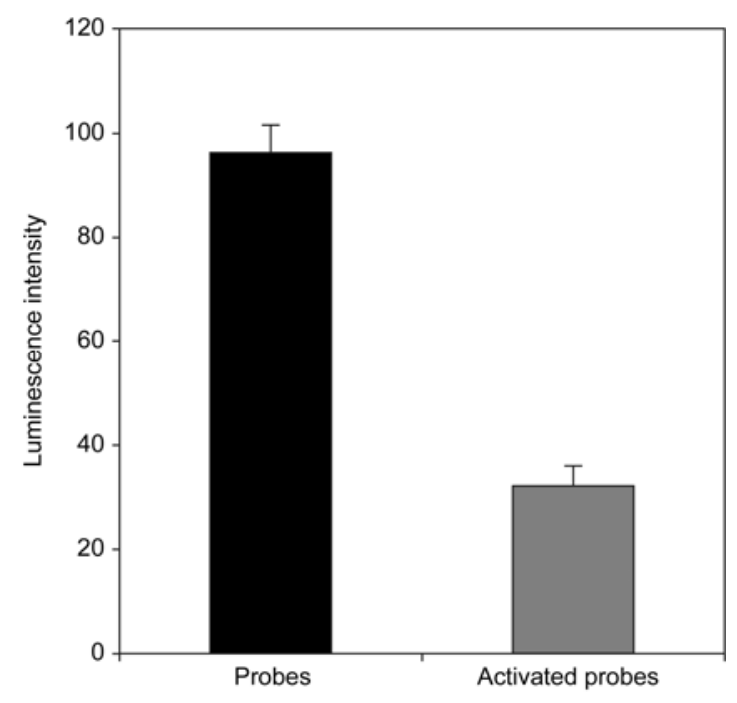

Figure 12 This chart demonstrates the reduction of the probe reacting with MMOL/LP enzyme solution. Fluorescence intensity at $585 \mathrm{~nm}$ was measured.

vated probe (probes within MCF-7 solution) indicated statistically insignificant changes in luminescence during the 
same time intervals.

Preliminary results demonstrate proof-of-principle that the QD-peptide-RB probes were highly promising agents not only for extending the limits of optical imaging by providing an inherent increase in the signal: background ratio, but also for monitoring protease activity which may provide information about the metastatic potential of a tumor.

\section{Conclusion}

On the basis of the above results, FRET between the CdTe QD and RB was successfully applied in sensing MMOL/ LPs with specific cleavage. When activated, a quenching of QD and an excitation of RB happened to the probe. With the further studies in the biology field, the donor-acceptor pair of QD and RB will exhibit more utilities and features in design of assays systems based on their interaction. Several parameters need further optimization for the advancement of clinically applicable QD protease probes, like inherent biocompatibility and the acceptor valence. An important issue in proteolytic activity is peptide accessibility, which is affected by interparticle distance between the QD and RB, the density of peptide-RB surrounding the QD. All of these parameters will be individually characterized to tailor reaction rates of proteolytic activation in future studies.

Current fluorescent imaging agents provide the increased contrast by accumulation of fluorophores with specific targets of interest. These agents supply limited functionality in the ability to observe molecular specific processes. Developing smart optical contrast agents that respond to molecular processes, for example protease activity, is pressing. The QD probe not only provides the advantages of observing time- or spatial-dependent processes, but also ensures an inherent increase in signal:background ratio greater than conventional targeted fluorophores. This nanosensor is flexible and can be fine-tuned for a wide array of applications such as early diagnosis of disease, the detection of biomarkers, and monitoring therapeutic efficacy simply by changing the sequence of the peptide linker. Future studies will be committed to optimizing and enhancing the quenching and unquenching efficiency of these smart probes. Moreover, due to the narrow emission bandwidth of QDs, it is potential to construct a library of different wavelength emitting biocompatible quenched probes, each with its own enzyme specificity and acceptor pair to allow for simultaneous imaging from many different proteases.

This work was supported by the National Natural Science Foundation of China (81000666, 81071194 and 81171395) and the Major Project of the
Ministry of Science and Technology for New Drug Development (2009ZX09310-004).

1 Aleksandra Z, Song D, Qian W, et al. Protein microarrays and quantum dot probes for early cancer detection. Coll Surfaces B: Biointerfaces, 2007, 58: 309-314

2 Dusica M, Jasmina L, Adi E, et al. Fate of micelles and quantum dots in cells. Eur J Pharma Biopharma, 2007, 65: 270-281

3 Wang Y, Chen L. Quantum dots, lighting up the research and development of nanomedicine. Nanomedicine, 2011, 7: 385-402

4 Richard J B, Elizabeth R H. Quantum dots brighten biological imaging. Prog Histochem Cytochem, 2011, 45: 201-237

5 Vasudevanpillai B, Sathish M, Remakrishnapillai V O, et al. Bioconjugated quantum dots for cancer research: Present status, prospects and remaining issues. Biotechnol Adv, 2010, 28: 199-213

6 Robert F H, Lauren A F, Mary F C, et al. Fluorescence resonance energy transfer analysis of merlin conformational changes. Mol Cell Biol, 2010, 30: 54-67

7 Li J, Lu C, Yao Q, et al. A graphene oxide platform for energy transfer-based detection of protease activity. Biosens Bioelectron, 2011, 26: 3894-3899

8 Deng S, Lei J, Cheng L, et al. Amplified electrochemiluminescence of quantum dots by electrochemically reduced graphene oxide for nanobiosensing of acetylcholine. Biosens Bioelectron, 2011, 26: 45524558

9 Vasudev J B, Brian P K, Christopher R R, et al. DNA methylation detection using MS-q FRET, a quantum dot-based nanoassay. Methods, 2010, 52: 237-241

10 Liang G, Pan H, Li Y, et al. Near infrared sensing based on fluorescence resonance energy transfer between Mn:CdTe quantum dots and Au nanorods. Biosens Bioelectron, 2009, 24: 3693-3697

11 Kai K, Vicki P, Zena W, et al. Matrix Metalloproteinases: Regulators of the tumor microenvironment. Cell, 2010, 141: 52-67

12 Rajeshwar P V, Corwin H. Matrix metalloproteinases (MMOL/LPs): Chemical-biological functions and (Q) SARs. Bioorg Med Chem, 2007, 15: 2223-2268

13 Michelle D M, Lynn M M. The other side of MMOL/LPs: Protective roles in tumor progression. Cancer Meta Rev, 2007, 26: 717-724

14 Malin S, Raghu K. Tumor stroma derived biomarkers in cancer. Cancer Meta Rev, 2009, 28: 177-183

15 Christoph B, Sebastian B, Umar M. Optical imaging of matrix metalloproteinase-Activity in tumors: Feasibility study in a mouse model. Radiology, 2001, 221: 523-529

16 Wang X, Lou X, Wang Y, et al. QDs-DNA nanosensor for the detection of hepatitis B virus DNA and the single-base mutants. Biosens Bioelectron, 2010, 25: 1934-1940

17 Tracey A M, Wen G J. Loss of tight junction barrier function and its role in cancer metastasis. Biochim Biophys Acta, 2009, 1788: 872891

18 Xue B, Deng D, Cao J, et al. Synthesis of NAC capped near infraredemitting CdTeS alloyed quantum dots and application for in vivo early tumor imaging. Dalton Transact, 2012, 41: 4935-4947

19 Asmae K, Pezhman M, Nadia B, et al. Matrix metalloproteinase-9 is required for tubular network formation and migration of resistant breast cancer cells MCF-7 through PKC and ERK1/2 signalling pathways. Cancer Lett, 2010, 295: 242-251

20 Sun Y, Lu N, Ling Y, et al. Oroxylin A suppresses invasion through down-regulating the expression of matrix metalloproteinase-2/9 in MDA-MB-435 human breast cancer cells. Eur J Pharmacol, 2009, 603: 22-28

21 Wu D, Chen Z. ZnS quantum dots as $\mathrm{pH}$ probes for study of enzyme reaction kinetics. Enzyme Microbial Technol, 2012, 51: 47-52

Open Access This article is distributed under the terms of the Creative Commons Attribution License which permits any use, distribution, and reproduction in any medium, provided the original author(s) and source are credited. 